\title{
General ultrafast pulse measurement using the cross-correlation single-shot sonogram technique
}

\author{
Derryck T. Reid and Jesus Garduno-Mejia \\ Ultrafast Optics Group, Department of Physics, School of Engineering and Physical Sciences, Heriot-Watt University, \\ Edinburgh EH14 4AS, Scotland, UK \\ Received September 12, 2003
}

\begin{abstract}
The cross-correlation single-shot sonogram technique offers exact pulse measurement and real-time pulse monitoring via an intuitive time-frequency trace whose shape and orientation directly indicate the spectral chirp of an ultrashort laser pulse. We demonstrate an algorithm that solves a fundamental limitation of the cross-correlation sonogram method, namely, that the time-gating operation is implemented using a replica of the measured pulse rather than the ideal delta-function-like pulse. Using a modified principal-components generalized projections algorithm, we experimentally show accurate pulse retrieval of an asymmetric double pulse, a case that is prone to systematic error when one is using the original sonogram retrieval algorithm. (C) 2004 Optical Society of America

OCIS codes: $320.7100,320.7160,190.7110$.
\end{abstract}

The cross-correlation sonogram (CCS) measures the dynamic spectrogram ${ }^{1}$ of ultrashort pulses and has been implemented using second-harmonic generation and two-photon cross correlation with a scanning-slit spectrometer $^{2,3}$ and Fabry-Perot filters. ${ }^{4,5}$ Recently we described a single-shot implementation for acquiring a CCS trace directly on a video camera ${ }^{6}$ that offers real-time intuitive pulse monitoring at video rates and uses a unique phase-matching geometry and tight focusing to achieve simultaneously the necessary cross-correlation and spectral filtering operations. Despite the many embodiments that are possible for implementing the spectral filtering step that is required in a sonogram measurement, the cross-correlation operation is the only practical way of implementing the accompanying time-gating step. When a source of narrow strobe pulses exists at the same repetition frequency as the pulses to be measured, the CCS technique yields a trace that is closely approximated by the formal sonogram signal, ${ }^{7}$

$$
I_{\text {sono }}(t, \Omega)=\left|\int_{-\infty}^{\infty} E(\omega) G(\omega-\Omega) \exp (i \omega t) \mathrm{d} \omega\right|^{2},
$$

which describes a pulse with a complex spectral amplitude $E(\omega)$ that is time gated in a way that records the group delay of each of its frequency components. This time gating is done by spectrally filtering $E(\omega)$ by use of a bandpass filter with an amplitude transmission profile $G(\omega)$ that can be detuned from the pulse center frequency by an arbitrary frequency $\Omega$. Analyses of sonogram measurements made with independent reference pulses were reported previously ${ }^{8,9}$ but when no separate strobe pulses exist the cross-correlation operation yields a trace that is no longer well described by Eq. (1) but is instead given by the expression

$$
I_{X-\text { sono }}(t, \Omega)=I(t) \otimes I_{\text {sono }}(t, \Omega) .
$$

where $\otimes$ is the convolution operation and $I(t)$ is the pulse intensity profile. The trace measured by use of cross correlation to implement the time-gating operation is properly described by Eq. (1) only when the measured pulse is single peaked and is readily broadened by the narrowband filtering action of the spectral filter used. Under these conditions, $I_{X \text {-sono }}(t, \Omega) \approx$ $I_{\text {sono }}(t, \Omega)$, but a significant deviation between the two forms of sonogram arises when the CCS of a double (or multiple) pulse is recorded. Like the autocorrelation, the CCS trace of a double pulse characteristically shows three maxima; however, the true sonogram trace given by Eq. (1) in fact exhibits only two maxima, one for each pulse. For a practical sonogram measurement it is important to find an algorithm that can invert a CCS trace. The double- (or multiple-) pulse case is affected worst by implementation of the time-gating step by use of cross correlation, but even measurements of single pulses made with cross correlation will generate sonograms that deviate from Eq. (1) if the bandwidth of the spectral filter used is too broad or the pulses are highly chirped. In the latter case the frequency-filtering operation has little effect on the pulse duration, and the unfiltered pulses no longer resemble short sampling functions with respect to the filtered pulses. In practice, this effect is negligible for the moderate chirp encountered when one is measuring pulses from a mode-locked laser oscillator.

To understand how the new algorithm accounts for the cross-correlation operation, consider first the original sonogram construction and inversion algorithm. In the standard construction procedure the trace is formed by creating the outer product between the initial guesses for the spectral amplitudes of the pulse, $E(\omega)$, and the filter function, $G(\omega)$, and then performing a matrix permutation operation that sorts the outer product matrix into rows of constant absolute frequency $\omega$ and columns of constant frequency detuning $\Omega$. The Fourier transform of the resulting matrix is the complex amplitude of the true sonogram trace, $I_{\text {sono }}(t, \Omega)$, and the phase retrieval proceeds by replacement of the intensity of this trace with that measured experimentally, $I_{\mathrm{lab}}(t, \Omega)$, and then use of principal-components generalized projections ${ }^{10}$ to extract improved estimates of the pulse and filter spectral amplitude from the modified trace. We have modified this algorithm to obtain retrieval 
that accounts for the corruption of the sonogram trace by the convolution with the pulse intensity, and the new algorithm is depicted schematically in Fig. 1. Instead of using the experimental trace in the intensity-replacement step of the algorithm, we first deconvolve it with the most recently obtained pulse intensity profile. When this procedure is implemented the first few iterations of the algorithm are less efficient and convergence is slower because the estimate of the pulse intensity, $I(t)$, is poor and noise can be introduced into an otherwise high-quality CCS trace. Often the noise introduced at this stage can be effectively controlled by windowing the resulting trace to enforce the condition of compact support that is generally required in the solution of two-dimensional phase-retrieval problems. ${ }^{11}$ As the estimate of $I(t)$ improves, the convergence becomes more rapid and the algorithm recovers the actual pulse, despite the absence of the true sonogram trace in the input data. During execution a measure of the algorithm convergence is given by rms error $G$ (see Fig. 1) between the normalized CCS used as input data and the normalized CCS synthesized on each iteration of the algorithm with the current estimate of the pulse and spectral gate. The algorithm can be run for an indefinite period until the rms error has fallen below a chosen threshold value, but in practice we find that 100-200 iterations is often sufficient, and we select the pulse and filter pair with the smallest rms value and output these after a predetermined number of iterations. We found that the solution was stable even after several thousand further iterations.

We illustrate the effectiveness of the algorithm for synthetic data and for data measured with a single-shot sonogram system. The synthetic data was a 256 -element asymmetric chirped double pulse. Figure 2 illustrates the difference between the synthesized CCS trace used to simulate an experimental trace [Fig. 2(a)] and the true sonogram [Fig. 2(b)]. Using 200 iterations of the modified algorithm, we correctly retrieved the true sonogram trace [Fig. 2(c)] and recovered the correct pulse shape [Fig. 3(a)]. When the original sonogram retrieval algorithm was applied, it failed to correctly retrieve the true sonogram trace and yielded pulse data that were very different from the correct shape [Fig. 3(b)].

The modified retrieval algorithm was next applied to experimental data obtained from a single-shot sonogram instrument used to measure 850-nm pulses from a self-mode-locked Ti:sapphire laser oscillator that had been chirped by an SF-10 prism pair (apex separation, $\sim 70 \mathrm{~cm}$ ) and then split to form a double pulse comprising two pulses of similar profiles but different peak intensities. Figure 4 depicts the CCS trace measured with the single-shot sonogram instrument [Fig. 4(a)] along with the true sonogram trace [Fig. 4(b)] inferred from the pulse data retrieved with Eq. (1) and the CCS trace [Fig. 4(c)] calculated with Eq. (2). The results of a retrieval after 150 iterations are presented in Fig. 5, and the retrieved pulse intensity and phase appear in Fig. 5(a). The retrieval indicates a strong pulse $(t \sim 0 \mathrm{fs})$ with a duration of $82 \mathrm{fs}$ and a weaker delayed pulse $(t \sim 750 \mathrm{fs})$ with a duration of $84 \mathrm{fs}$. The two pulses were expected to have slightly different durations because the delayed pulse experienced a double pass through a 1-mm beam-splitter substrate, incurring some additional chirp. A weak artifact of the retrieval algorithm is present at $t \sim-750 \mathrm{fs}$ and is attributed to experimental noise, and a slight nonlinear skew of the experimental trace used as the input to the retrieval algorithm (linear skew or trace rotation is easily removed numerically and if left uncorrected is a significant source of systematic error). Figure 5(b) shows the retrieved spectrum (dotted curve with symbols) that accurately reproduces the rapid modulation observed with a separate spectrometer [Fig. 5(b) solid curve]. As well as closely reproducing the period of the spectral fringes, the retrieved spectrum matches

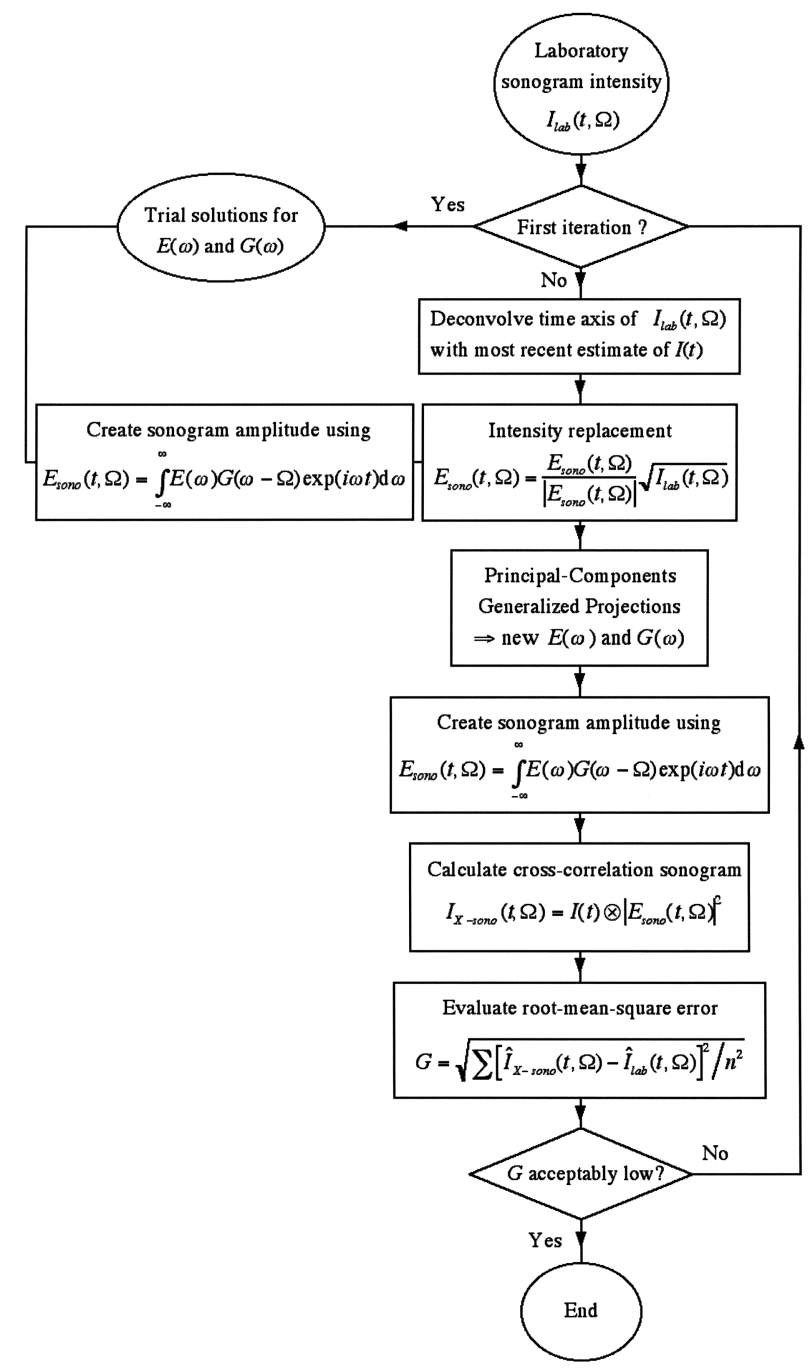

Fig. 1. Modified CCS phase-retrieval algorithm.
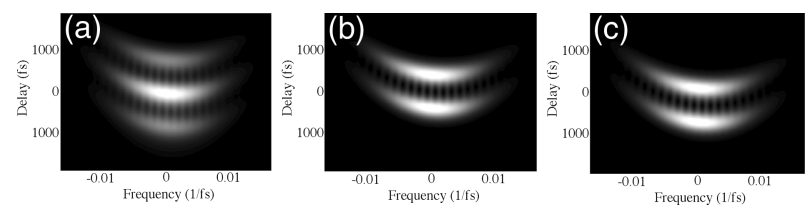

Fig. 2. (a) Simulated CCS used as input data for the new algorithm. (b) True sonogram of the input pulses generated using Eq. (1). (c) Sonogram retrieved with the new algorithm. 

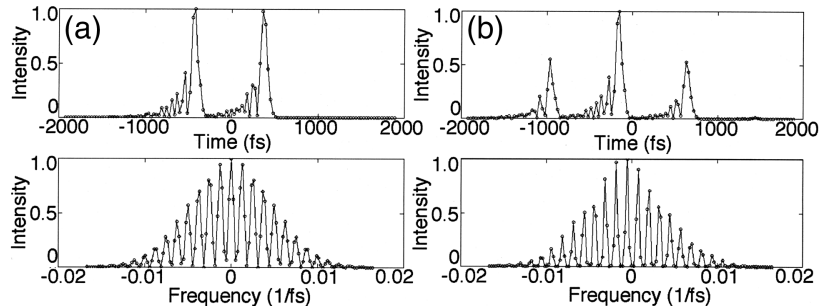

Fig. 3. Pulse temporal (top) and spectral (bottom) profiles: (a) pulses used as the test data and also correctly retrieved by the new sonogram retrieval algorithm (the test data and retrieved data are identical), (b) pulses incorrectly retrieved with the original sonogram retrieval algorithm.
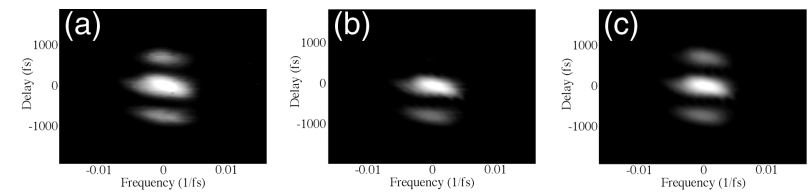

Fig. 4. (a) Measured CCS trace, (b) retrieved sonogram trace, (c) CCS trace calculated from the retrieved pulse data.
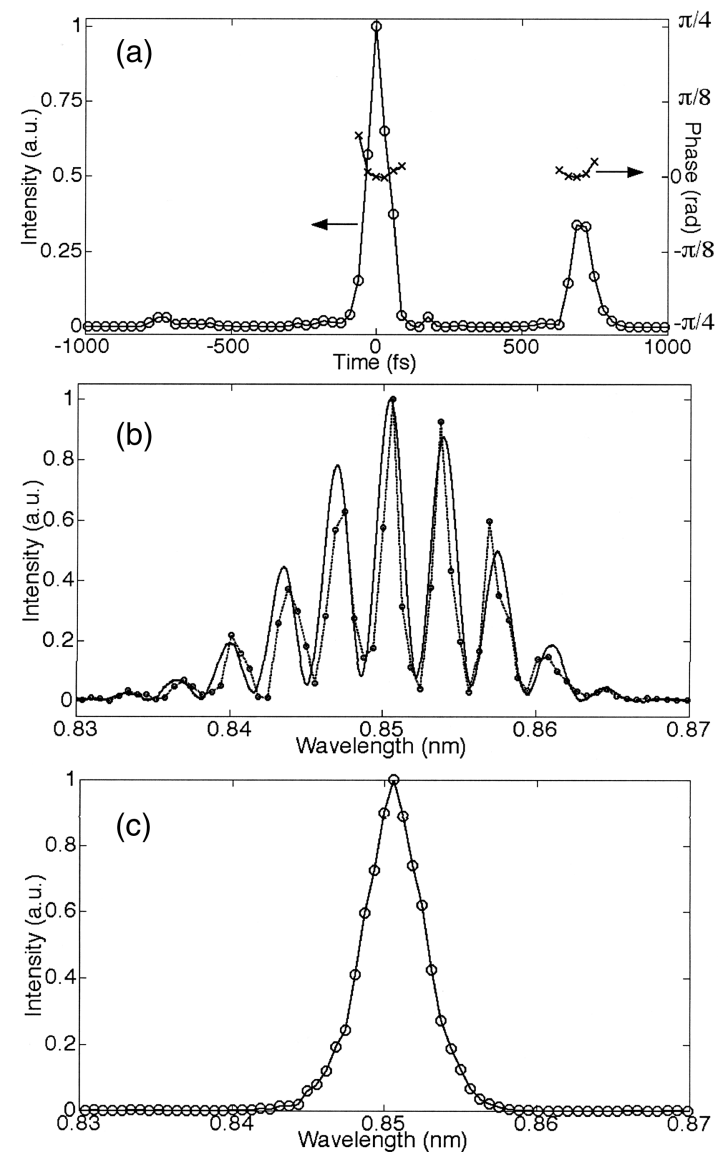

Fig. 5. (a) Retrieved double pulse showing the difference in the pulse intensities, (b) retrieved pulse spectrum (symbols and dotted curve) with the independently measured spectrum (solid curve), (c) retrieved spectral filter.

the modulation depth of the independently measured spectrogram, meaning that the relative intensities of the pulses recovered by the algorithm are correct. This result is impressive because the spectral resolution of the filter function used to record the sonogram trace [Fig. 5(c)] is itself too coarse to resolve the spectral modulation directly on the trace. This paradox is explained because the frequency resolution of the sonogram method is determined, as in other time-frequency techniques, by the delay span and the sampling period as well as the resolution of the filter used in the trace acquisition. Indeed, for a practical measurement it is often disadvantageous to use a filter with a spectral resolution much finer than the pulse bandwidth because this results in a sonogram trace with a temporal width that is much greater than the pulse itself. For the single-shot sonogram configuration used here this criterion means that a relatively short nonlinear crystal is desirable, and the results shown here were obtained with a 2-mm-thick KDP crystal. In pulse complexity terms, the single-shot sonogram instrument should be able to measure a pulse chirped to 5-10 times its transform-limited duration.

The ability of the modified algorithm to retrieve experimental data accurately is highly significant for the sonogram technique because, for what is to our knowledge the first time, the CCS method (the simplest to implement experimentally) is not restricted to measuring only simple pulse shapes but can be used to effectively measure highly structured pulses containing multiple peaks and complex chirp. The retrieved data resulted in a rms error of $2.4 \%$ between the measured and the synthetic CCS traces, and in a sonogram retrieval this magnitude typically corresponds to good retrieval. With this limitation removed, the sonogram approach is competitive with the second-harmonic-generation frequency-resolved optical gating method, which is also capable of measuring highly structured pulses but, unlike in SHG frequency-resolved optical gating, the CCS is capable of directly measuring both the sign and the magnitude of the frequency chirp on the pulses.

The authors gratefully acknowledge the support of the Paul Instrument Fund for this work. D. T. Reid's e-mail address is d.t.reid@hw.ac.uk.

\section{References}

1. E. B. Treacy, J. Appl. Phys. 42, 3848 (1971).

2. V. Wong and I. A. Walmsley, J. Opt. Soc. Am. B 14, 944 (1997).

3. I. G. Cormack, W. Sibbett, and D. T. Reid, J. Opt. Soc. Am. B 18, 1377 (2001).

4. I. G. Cormack, W. Sibbett, R. Ortega-Martinez, and D. T. Reid, Rev. Sci. Instrum. 72, 4071 (2001).

5. D. T. Reid, B. C. Thomsen, J. M. Dudley, and J. D. Harvey, Electron. Lett. 36, 1141 (2000).

6. D. T. Reid and I. G. Cormack, Opt. Lett. 27, 658 (2002).

7. D. T. Reid, IEEE J. Quantum Electron. 35, 1584 (1999).

8. K. Kikuchi and K. Taira, IEEE J. Quantum Electron. 37, 533 (2001).

9. K. Taira and K. Kikuchi, IEEE Photon. Technol. Lett. 13, 505 (2001).

10. D. J. Kane, IEEE J. Sel. Top. Quantum Electron. 14, 944 (1998).

11. H. V. Deighton, M. S. Scivier, and M. A. Fiddy, Opt. Lett. 10, 250 (1985). 